\title{
Pseudomonas bacteriophage AN14 - a Baikal-borne representative of Yuavirus
}

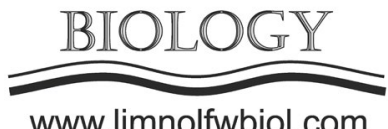

www.limnolfwbiol.com

\author{
Evseev P.V. ${ }^{1}$, Gorshkova A.S. ${ }^{2}$, Sykilinda N.N. ${ }^{1}$, Drucker V.V. ${ }^{2}$, Miroshnikov K.A. ${ }^{1 *}$ \\ ${ }^{1}$ Shemyakin-Ovchinnikov Institute of Bioorganic Chemistry, Russian Academy of Sciences, Miklukho-Maklaya Str., 16/10, Moscow, \\ 117997, Russia \\ ${ }^{2}$ Limnological Institute, Siberian Branch of the Russian Academy of Sciences, Ulan-Batorskaya Str., 3, Irkutsk, 664033, Russia
}

\begin{abstract}
Siphoviruses with a prolate capsid infecting Pseudomonas sp. were isolated from environmental sources through the world, and were recently defined as a separate taxonomic genus Yuavirus. Besides a distinguishable morphology, the hallmarks of the genus are heavily modified genomic DNA and a lytic infection cycle while putative lysogeny genes are proposed in the genomes. Bacteriophage AN14 was isolated from Lake Baikal ecosystem and was assigned as a member of Yuavirus. We report the biological and morphological features if this phage, as well as the comprehensive reannotation of its genome. Modern bioinformatics and phylogenomic analysis resulted in the refinement of the taxonomic attribution of the YuA-like phages and highlighting the specific genomic and proteomic features typical for Yuavirus phages including AN14.
\end{abstract}

Keywords: Bacteriophage, Pseudomonas, genomics, phylogeny, taxonomy

\section{Introduction}

Bacteriophage AN14 infecting Pseudomonas was discovered in the aquatic ecosystem of Lake Baikal in 2010. According to the current nomenclature justified by the International Committee for Virus (ICTV) in 2020, phage AN14 belongs to the genus Yuavirus of family Siphoviridae. ICTV database lists six previously reported phages as members of Yuavirus - Alphaproteobacteria virus $\varphi J L 001$, and Pseudomonas viruses LKO4, M6, MP1412, PAE1 and YuA. Moreover, the NCBI database and taxonomic browser contains information on another 12 phages presumably belonging to the genus Yuavirus. Most of them infect Pseudomonas sp., however the set includes also a Bordetella phage LK3. Previously, other Bordetella phages (CN1, CN2, FP1 and MW2) were considered as members of Yuavirus. These viruses are currently attributed as a separate genus Vojvodinavirus. The representatives of Yuavirus phages were found in geographically distant environmental aqueous reservoirs. Extreme diversity of the bacterial hosts of this phage group comprising alpha-, beta- and gammaproteobacteria raises questions on the correction and possible revision of the current taxonomic classification.

The presented work reports the parameters of the AN14 infection cycle and morphology. We have re-annotated the AN14 genome taking into account the recent data on structures and functions of phage proteins. Genomic and phylogenetic research has refined the taxonomic position of phages belonging to genus Yuavirus.

\section{Materials and methods}

\subsection{Phage propagation and purification}

Pseudomonas aeruginosa strain PAO1 (ATCC 15692), purchased from the American Type Culture Collection (ATCC), was used as a host for phage propagation. A water sample taken from Selenga river (52.090687 106.637384) (Fig. 1) (3 mL) was supplemented with $1 \mathrm{~mL}$ of $4 \times$ lysogeny broth (LB) and $40 \mu \mathrm{L}$ overnight culture of $\mathrm{PAO} 1$, and incubated at $37^{\circ} \mathrm{C}$ for $18 \mathrm{~h}$. Chloroform was added to a final concentration of $0.5 \%(\mathrm{v} / \mathrm{v})$ for $4 \mathrm{~h}$ at $4^{\circ} \mathrm{C}$. The suspension was then centrifuged at $7000 \times \mathrm{g}$ for $20 \mathrm{~min}$. The presence of bacteriophages and the titer in the supernatant was determined by the appearance of plaques on the bacterial lawn using the double-agar layer technique (Adams, 1959) with minor modifications. The phage from a single plaque was propagated using a liquid culture of the $P$. aeruginosa PAO1 host strain. The incubation was performed at $37^{\circ} \mathrm{C}$ until the lysis completion, and then chloroform was added. Bacterial debris was pelleted by centrifugation at $3000 \times \mathrm{g}$ for $20 \mathrm{~min}$. The phage lysate was precipitated with polyethylene glycol (PEG) $8000(10 \%)-\mathrm{NaCl}(0.6 \%)$ at $4^{\circ} \mathrm{C}$ overnight, centrifuged at $8000 \times \mathrm{g}$ for $20 \mathrm{~min}$ at $4^{\circ} \mathrm{C}$, resuspended in the SM buffer $(50 \mathrm{mM}$ Tris- $\mathrm{HCl}(\mathrm{pH}$ 7.5), $100 \mathrm{mM} \mathrm{NaCl}, 8 \mathrm{mM} \mathrm{MgSO}, 0.01 \%$ gelatin) and then $1 \mathrm{M} \mathrm{KCl}$ was added. The mixture was incubated on ice for $20 \mathrm{~min}$ and centrifuged $(12000 \times \mathrm{g}$ for 20 min at $4{ }^{\circ} \mathrm{C}$ ) to precipitate PEG (Colombet et al., 2007). The phage preparation was purified by cesium chloride 
equilibrium gradient centrifugation at $22000 \times \mathrm{g}$ (Beckman SW41 Ti rotor, Germany) for $2 \mathrm{~h}$. The phage band was collected and dialyzed overnight against 0.01 $\mathrm{M}$ Tris- $\mathrm{HCl}$ (pH 7.5), $0.01 \mathrm{M} \mathrm{MgSO}_{4}, 0.15 \mathrm{M} \mathrm{NaCl}$ at $4^{\circ} \mathrm{C}$. The titer of the resulting phage was about $10^{11}$ plaque forming units (PFU)/mL.

\subsection{Phage one-step growth curve}

Phage suspension was mixed with $1 \mathrm{~mL}$ of exponential phase culture of strain PAO1 at $\mathrm{MOI}=0.1$ and incubated at $37^{\circ} \mathrm{C}$ for $10 \mathrm{~min}$ for phage adsorption. The mixture was centrifuged at $10,000 \times \mathrm{g}$ for $5 \mathrm{~min}$ to remove free phage particles. The pellet was resuspended in $50 \mathrm{~mL}$ of $\mathrm{LB}$, and the culture was continuously incubated at $37^{\circ} \mathrm{C}$. Samples were taken at $10 \mathrm{~min}$ intervals to $2 \mathrm{~h}$, and phage titer was determined.

\subsection{Electron microscopy}

The morphology of phage AN14 was examined by negative strain transmission electron microscopy (TEM). Briefly, a single plaque was resuspended in 100 $\mu \mathrm{L}$ of saline $(0.8 \% \mathrm{NaCl}$ aqueous solution), centrifuged for $15 \mathrm{~min}$ at $4000 \times \mathrm{g}$, and the supernatant was filtered through a $0.22 \mu \mathrm{m}$ filter. The prepared phages were placed on freshly-prepared formvar films with $2 \%(\mathrm{w} / \mathrm{v})$ uranyl acetate. Morphological characteristics were observed under a transmission electron microscope (Zeiss LEO 906E, Germany).

\subsection{Reannotation of phage genome}

The previously deposited genomic sequence of phage AN14 was downloaded from GenBank (Accession number KX198613) and annotated by predicting and validating open reading frames (ORFs) using Prodigal 2.6.1 (Hyatt et al., 2010) and Prokka 1.17 (Seemann, 2014) pipelines. Identified ORFs were manually curated to ensure fidelity. Functions were assigned to ORFs using a BLAST search on a custom phage protein database compiled from annotated phage GenBank sequences, InterPro server (https://www.ebi.ac.uk/ interpro/entry/InterPro) and HHpred server (https:// toolkit.tuebingen.mpg.de) with Pfam-A_v32.0, NCBI Consreved_Domain_v.3.16, SMART_v6.0, PRK_6.9, PDB, SCOPe70_2.07, ECOD_ECOD_F70_20190225 and COG_KOG_v1.0 databases. Custom BLAST databases were mounted with the BLAST tool (https://blast. ncbi.nlm.nih.gov/Blast.cgi). tRNA coding regions were searched with tRNAscan-SE (Schattner et al., 2005). The resulting genome map was visualized in Geneious Prime, version 2020.0.5 (https://www.geneious.com).

\subsection{Phylogenetic analysis}

Phage reference genomes were downloaded from NCBI GenBank (ftp://ftp.ncbi.nlm.nih.gov/genbank). Whether necessary, the genomes were annotated using Prokka (Seemann, 2014), with a custom phage protein database compiled from annotated phage GenBank

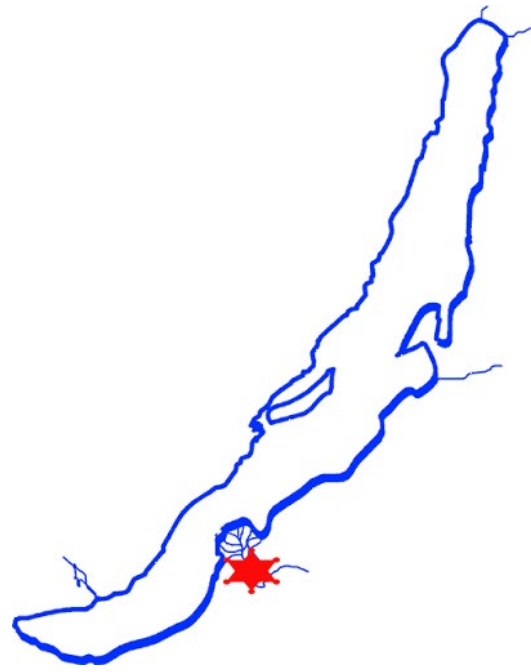

Fig.1. Map of Lake Baikal. The sampling station is indicated by an asterisk.

sequences. A search for homologous sequences was conducted using a BLAST search and found sequences were checked for the presence of annotated homologous genes in NCBI genomes. Genes were extracted from GenBank annotations. For some unannotated sequences, ORFs were found by Glimmer3 (Delcher et al., 1999). ORFs were validated and corrected by comparison with known homologous genes. Protein alignments were made with MAFFT (Katoh et al., 2002) (L-INS-i algorithm, BLOSUM62 scoring matrix, 1.53 gap open penalty, 0.123 offset value). The alignments were trimmed manually and with trimAL (Capella-Gutiérrez et al., 2009) with gappyout settings. Best protein models were found with MEGAX 10.0.5 (Kumar et al., 1994). Phylograms were generated based on the amino acid sequences of proteins and their concatenated alignments, using Geneious Prime and MAFFT for sequence alignment. Trees were constructed using the maximum likelihood (ML) method with an RAxML program (Stamatakis, 2014) with a WAG + G protein model and the robustness of the trees was assessed by bootstrapping (1000).

\subsection{Whole-genome and proteome analysis}

Average nucleotide identity (ANI) was computed using the OrthoANIu tool (Lee et al., 2016), employing USEARCH (http://www.drive5.com/usearch/) over BLAST (https://www.ezbiocloud.net/tools/orthoaniu) with default settings and with an EzBioCloud server (https://www.ezbiocloud.net/tools/ani). Genome comparison was made with BRIG (Alikhan et al., 2011). Proteome analysis was performed with BPGA software (Chaudhari et al., 2016).

\subsection{D homology modeling, alignment and visualization}

Protein remote homology detection, 3D structure prediction and template-based homology prediction were made by Phyre 2 protein fold recognition server (Kelley et al., 2015) (http://www.sbg.bio.ic.ac. 
uk/ phyre2), HHpred (https://toolkit.tuebingen. mpg.de/tools/hhpred). The obtained structures were aligned and visualized with UCSF Chimera (Goddard et al., 2018).

\section{Results and discussion}

\subsection{General biological characteristics of phage AN14}

Bacteriophage AN14 was isolated and propagated using the type strain PAO1 of Pseudomonas aeruginosa. It produces small clear plagues $(\sim 1 \mathrm{~mm}$ diameter). The one-step growth curve shows a latent period of $40 \pm$ $5 \mathrm{~min}$, slow lysis, and a burst size about $110 \mathrm{PFU} /$ cell at $37^{\circ} \mathrm{C}$ (Fig. 2). The infection range of phage AN14 is considered as relatively narrow. Only two of 53 assayed environmental and clinical isolates of $P$. aeruginosa were susceptible to AN14.

Electron microscopic imaging reveals AN14 as a typical member of the Siphoviridae family (Fig. 3), having a flexible, non-contractile tail $(\sim 150 \mathrm{~nm})$ and an elongated capsid ( $\sim 70 \times 50 \mathrm{~nm}$ ) (B2 morphotype). These dimensions are typical for all representatives of Yuavirus where the morphology was recorded.

\subsection{AN14 genome - general features}

The number of entries in Genbank phage database grows rapidly (Sayers et al., 2019), as well as the number of resolved protein structures in the corresponding databases (https://www.rcsb.org/stats/ growth/growth-released-structures). Exploiting the recent available data enables us to improve the quality of existing annotations.

The length of phage AN14 dsDNA genome is 60,973 bp (accession number KX198613), and it exists in a circular permuted form. Therefore, the selection of the "zero point" is arbitrary, and a number of alternative ways of gene numeration are offered in GenBank annotations. We have chosen to align the AN14 genome with the genome of a type phage YuA, since it logically reflects the employment of genes in

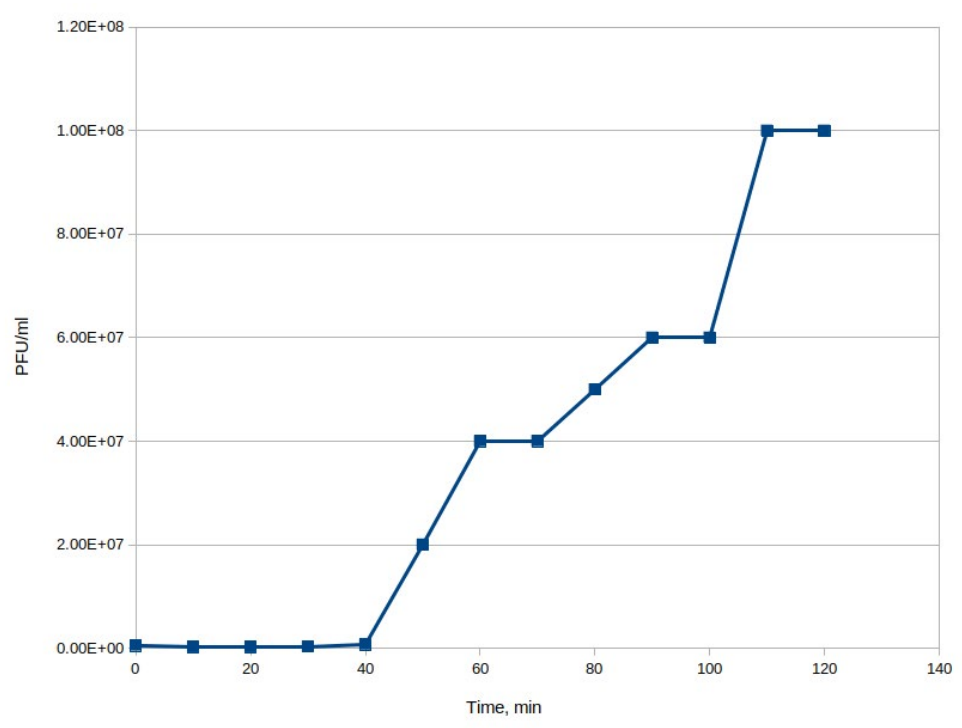

Fig.2. One step growth curve of AN14 using P. aeruginosa PAO1 as a host.

the infection cycle. The genome of AN14 (Fig. 4, Table 1) contains 86 putative ORFs, putative functions of 58 proteins can be predicted, and 28 ORFs are assigned as hypothetical proteins. There are no tRNA genes found in the genome. All genes are oriented in the same direction. G $+\mathrm{C}$ content of the AN14 genome is $64.5 \%$, evenly distributed through the genome (Fig. 4). Yuavirus phages infecting Pseudomonas have the $\mathrm{G}+\mathrm{C}$ value most close to the average $\mathrm{G}+\mathrm{C}(65 \%)$ of the bacterial host. This may be the trait of a long-term adaptation of the phage to its host (Ceyssens et al., 2008). Notably, the $\mathrm{G}+\mathrm{C}$ content of the genomes of Pseudomonas YuA-like phages (64.3\%-64.7\%) is higher than in the genomes of Alphaprotebacteria phage $\varphi \mathrm{JL001}$ (62\%) and Bordetella phage LK3 (64.0\%).

The genes of AN14 genome are clustered in three blocks - the first one encodes structural and lysis proteins, the second one is responsible for nucleotide metabolism and modification proteins, and the third block encodes replication, transcription and other proteins. The genes of structural proteins comprise about one third of the genome and are well conserved in

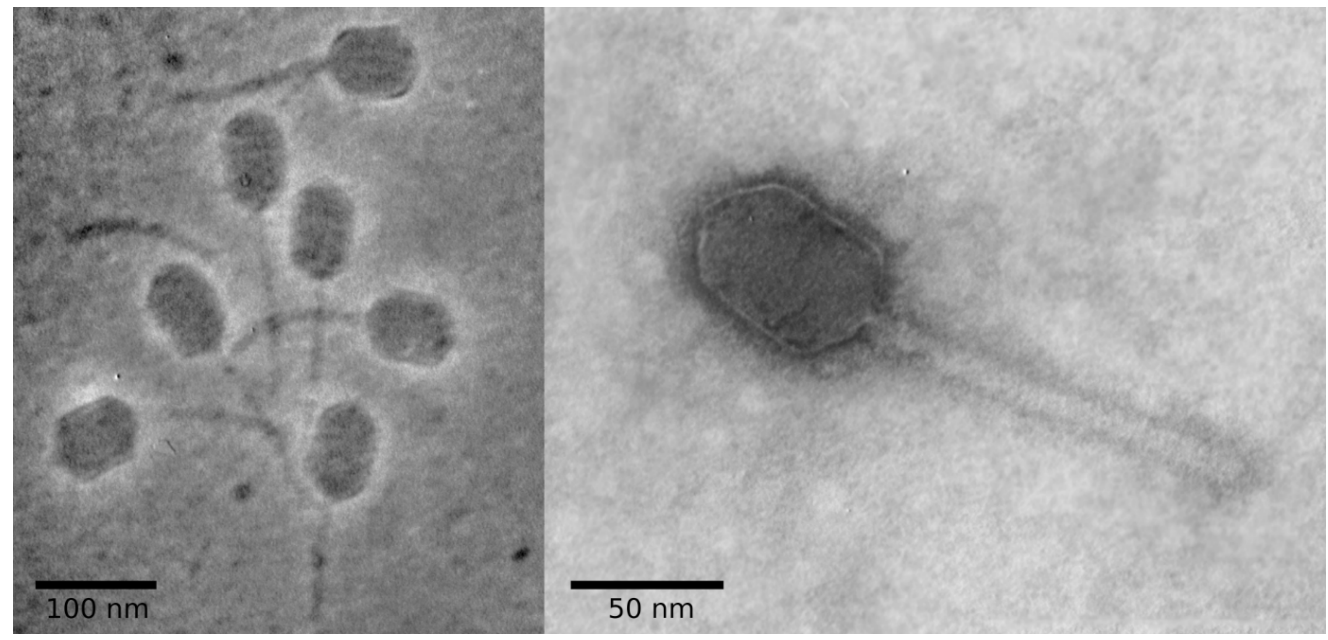

Fig.3. Transmission electron microscopy image of phage AN14. Staining with $2 \%$ uranyl acetate. Scale bars $-50 \mathrm{~nm}$ and $100 \mathrm{~nm}$. 
Table 1. Functional assignments of Pseudomonas phage AN14 genes.

\begin{tabular}{|c|c|c|c|c|c|}
\hline Name & From & To & Length & Direction & locus tag \\
\hline helicase & 379 & 2073 & 1695 & forward & gp01 \\
\hline hypothetical protein & 2081 & 2434 & 354 & forward & gp02 \\
\hline exonuclease inhibitor protein & 2494 & 3039 & 546 & forward & gp03 \\
\hline ATP-binding protein & 3182 & 4021 & 840 & forward & gp04 \\
\hline exodeoxyribonuclease V subunit RecD & 4123 & 5316 & 1194 & forward & gp05 \\
\hline ribonucleotide reductase & 5377 & 7200 & 1824 & forward & gp06 \\
\hline uracil-DNA glycosylase & 7342 & 7848 & 507 & forward & gp07 \\
\hline hypothetical protein & 7948 & 8631 & 684 & forward & gp08 \\
\hline hypothetical protein & 8642 & 8905 & 264 & forward & gp09 \\
\hline $\begin{array}{l}\text { alpha-glutamyl/putrescinyl thymine } \\
\text { pyrophosphorylase clade } 3\end{array}$ & 8915 & 9790 & 876 & forward & gp10 \\
\hline 1-aminocyclopropane-1-carboxylate deaminase & 9792 & 10628 & 837 & forward & gp11 \\
\hline radical SAM protein & 10700 & 12142 & 1443 & forward & gp12 \\
\hline thymidylate kinase & 12197 & 12760 & 564 & forward & gp13 \\
\hline $\begin{array}{l}\text { alpha-glutamyl/putrescinyl thymine } \\
\text { pyrophosphorylase clade } 1\end{array}$ & 12772 & 13743 & 972 & forward & gp14 \\
\hline hypothetical protein & 13799 & 14359 & 561 & forward & gp15 \\
\hline hypothetical protein & 14372 & 14485 & 114 & forward & gp16 \\
\hline thymidylate synthase/dCMP hydroxymethylase & 14482 & 15537 & 1056 & forward & gp17 \\
\hline putative phosphohydrolase & 15550 & 16185 & 636 & forward & gp18 \\
\hline hypothetical protein & 16160 & 16387 & 228 & forward & gp19 \\
\hline hypothetical protein & 16356 & 16742 & 387 & forward & gp20 \\
\hline DNA polymerase A & 16742 & 18736 & 1995 & forward & gp21 \\
\hline hypothetical protein & 18733 & 19047 & 315 & forward & gp22 \\
\hline phage_holin_1-like protein & 19044 & 19238 & 195 & forward & gp23 \\
\hline deoxycytidylate deaminase & 19235 & 19696 & 462 & forward & gp24 \\
\hline restriction endonuclease & 19689 & 20087 & 399 & forward & gp25 \\
\hline lambda-repressor-like protein & 20072 & 20398 & 327 & forward & gp26 \\
\hline DNA-primase & 20411 & 22822 & 2412 & forward & gp27 \\
\hline dsRBD-like protein & 22833 & 23048 & 216 & forward & gp28 \\
\hline membrane protein & 23160 & 23525 & 366 & forward & gp29 \\
\hline hydrolase, metallopeptidase & 23564 & 24742 & 1179 & forward & gp30 \\
\hline hypothetical protein & 24739 & 25080 & 342 & forward & gp31 \\
\hline hypothetical protein & 25092 & 25361 & 270 & forward & gp32 \\
\hline nucleotide pyrophosphohydrolase & 25358 & 25969 & 612 & forward & gp33 \\
\hline hypothetical protein & 25966 & 26442 & 477 & forward & gp34 \\
\hline hypothetical protein & 26439 & 26768 & 330 & forward & gp35 \\
\hline hypothetical protein & 26787 & 27083 & 297 & forward & gp36 \\
\hline hypothetical protein & 27086 & 27373 & 288 & forward & gp37 \\
\hline hypothetical protein & 27378 & 27647 & 270 & forward & gp38 \\
\hline hypothetical protein & 27644 & 27808 & 165 & forward & gp39 \\
\hline serine/threonine-protein kinase & 27787 & 28404 & 618 & forward & gp40 \\
\hline all-alpha NTP pyrophosphatase-like protein & 28431 & 29114 & 684 & forward & gp41 \\
\hline hypothetical protein & 29114 & 29395 & 282 & forward & gp42 \\
\hline
\end{tabular}




\begin{tabular}{|c|c|c|c|c|c|}
\hline Name & From & To & Length & Direction & locus tag \\
\hline 3'-5' exonuclease & 29397 & 29969 & 573 & forward & gp43 \\
\hline DNA-directed RNA polymerase subunit alpha & 29962 & 30153 & 192 & forward & gp44 \\
\hline hypothetical protein & 30254 & 30862 & 609 & forward & gp45 \\
\hline hypothetical protein & 30991 & 31254 & 264 & forward & gp46 \\
\hline DNA binding protein & 31306 & 32193 & 888 & forward & gp47 \\
\hline diguanylate-cyclase GGDEF-like protein & 32198 & 32668 & 471 & forward & gp48 \\
\hline antirestriction protein & 32763 & 33176 & 414 & forward & gp49 \\
\hline hypothetical protein & 33234 & 33563 & 330 & forward & gp50 \\
\hline hypothetical protein & 33586 & 33798 & 213 & forward & gp51 \\
\hline hypothetical protein & 33795 & 34190 & 396 & forward & gp52 \\
\hline hypothetical protein & 34187 & 34489 & 303 & forward & gp53 \\
\hline hypothetical protein & 34486 & 34815 & 330 & forward & gp54 \\
\hline hypothetical protein & 34812 & 35000 & 189 & forward & gp55 \\
\hline hypothetical protein & 35004 & 35255 & 252 & forward & gp56 \\
\hline terminase small subunit & 35924 & 36451 & 528 & forward & gp57 \\
\hline terminase large subunit & 36451 & 37998 & 1548 & forward & gp58 \\
\hline portal protein & 38056 & 39594 & 1539 & forward & gp59 \\
\hline membrane protein & 39591 & 39704 & 114 & forward & gp60 \\
\hline minor capsid protein & 39706 & 40914 & 1209 & forward & gp61 \\
\hline minor structural protein & 40983 & 41765 & 783 & forward & gp62 \\
\hline major capsid protein & 41961 & 42893 & 933 & forward & gp63 \\
\hline hypothetical protein & 43000 & 43605 & 606 & forward & gp64 \\
\hline hypothetical protein & 43633 & 43803 & 171 & forward & gp65 \\
\hline Rz-like lysis protein & 43807 & 44376 & 570 & forward & gp66 \\
\hline Rz1-like protein & 44039 & 44305 & 267 & forward & gp67 \\
\hline holin & 44373 & 44708 & 336 & forward & gp68 \\
\hline lysozyme, lytic murein transglycosylase $\mathrm{F}$ & 44693 & 45418 & 726 & forward & gp69 \\
\hline capsid decoration protein & 45418 & 45936 & 519 & forward & gp70 \\
\hline head-tail attachment protein & 45940 & 46308 & 369 & forward & gp71 \\
\hline tail protein & 46305 & 46757 & 453 & forward & gp72 \\
\hline major tail tube protein & 46791 & 48323 & 1533 & forward & gp73 \\
\hline tail assembly chaperone protein & 48386 & 48844 & 459 & forward & gp74 \\
\hline tail assembly chaperone protein & 48868 & 49134 & 267 & forward & gp75 \\
\hline putative tail competion protein & 49118 & 49564 & 447 & forward & gp76 \\
\hline tail tape measure protein & 49567 & 52392 & 2826 & forward & gp77 \\
\hline putative tail fiber protein & 52402 & 53931 & 1530 & forward & gp78 \\
\hline structural protein & 53946 & 54944 & 999 & forward & gp79 \\
\hline tail assembly structural protein & 54946 & 56616 & 1671 & forward & gp80 \\
\hline tail assembly structural protein & 56613 & 57422 & 810 & forward & gp81 \\
\hline membrane protein & 57436 & 57669 & 234 & forward & gp82 \\
\hline structural protein & 57669 & 57872 & 204 & forward & gp83 \\
\hline tail component & 57856 & 60027 & 2172 & forward & gp84 \\
\hline structural protein & 60027 & 60512 & 486 & forward & gp85 \\
\hline membrane protein & 60580 & 60924 & 345 & forward & gp86 \\
\hline
\end{tabular}




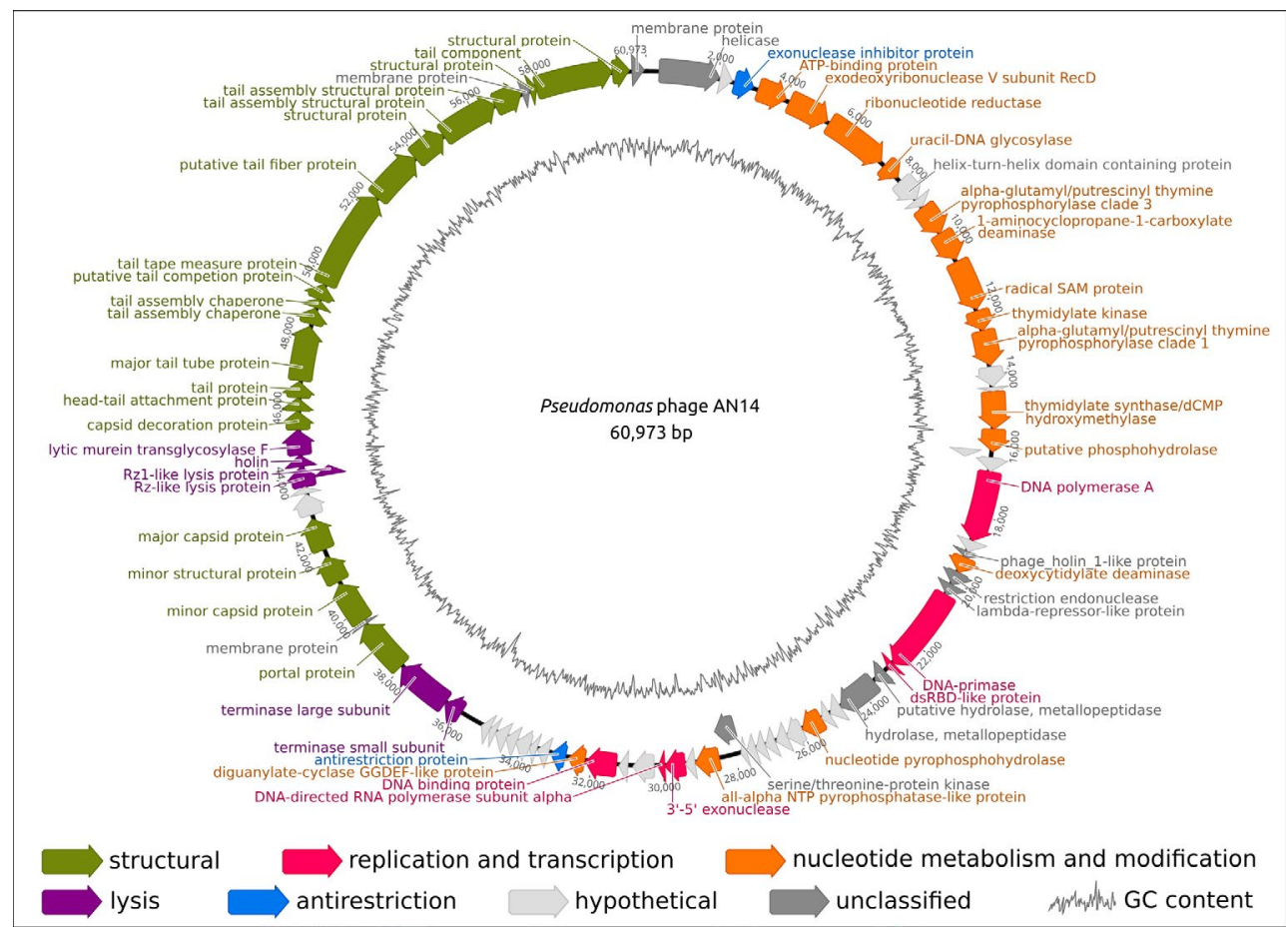

Fig.4. Circular diagram of functional assignments of the Pseudomonas phage AN14 genome. In total, 86 protein-coding genes are shown as colored blocks. The direction of transcription is shown by arrows. The GC content of the genome sequence is indicated by the internal grey curve.

the representatives of the genus. The functions of most of these genes can be predicted using BLAST and HMMHMM pipelines. A noticeable feature of phage YuA is the helical-shaped decoration of the prolate capsid with gene product (gp) 63 (Sokolova et al., 2010).The gene encoding the decoration protein is conservative among Yuavirus genomes and corresponds to g70 in AN14.

The block for terminal lysis of the host cell in Pseudomonas Yuavirus phages contains genes encoding peptidoglycan-lysing enzyme, which has been proposed to function as murein transglycosylase $\mathrm{F}$, and the holin located immediately downstream. The HMM-HMM and BLAST search also revealed the presence of nested genes encoding Rz-like and Rz1-like proteins adjacent to holin. Such proteins have been shown to form a complex spanning the periplasmic space, providing more efficient lysis of the host cell (Berry et al., 2008; Kongari et al., 2018). All these lysis proteins are conserved in the genomes of Yuavirus phages infecting Pseudomonas and Vojvodinavirus phages infecting Bordetella, but have no close homologs in Alphaprotebacteria phage $\varphi \mathrm{JL} 001$, which is misclassified, as we suggest. Therefore, we propose that the Yuavirus phages encode the combined lysis module involving endolysin, holin, and Rz-like/ Rz1-like proteins.

One of the noticeable features of AN14 predicted proteome is a significant number of proteins involved in nucleotide modification and repair. At least 8 genes for such proteins can be found, and their homologs are present in other Yuavirus genomes. A large set of potential nucleotide modification proteins correlates with the experimentally found high content of modified bases in the genomes of phages YuA (Ceyssens et al., 2008) and M6 (Lee et al., 2018). Another interesting feature of AN14 genome is the presence an anti-restriction system
(Spoerel et al., 1979), including antirestriction protein (gp49) and predicted exonuclease inhibitor protein (gp03). The AN14 genome contains diguanylatecyclase GGDEF domain protein (gp48). This protein can function as diguanylate cyclase (DGC) to form cyclic di-GMP, a global second messenger controlling bacterial motility and sessility. Higher activity of DGC results to an elevated cyclic di-GMP level plays an important role in the early stage of biofilm formation in P. aeruginosa (Simm et al., 2004; Bae et al., 2012). We have also identified genes presumably participating in amino acid metabolism such as serine/threonineprotein kinase (gp13). The previous annotation of AN14 genome and the annotations of the genomes of YuA-like phages genomes have stated a presence of an integrase-like protein (gp27), but we propose that this protein is most likely a DNA primase. Thus, we can reinterpret important features of the lifecycle of Yuavirus phages (see below).

\subsection{Taxonomy}

Calculations of average nucleotide identity (ANI) between AN14 and all 21067 phage sequences deposited in the NCBI GenBank (up to July 2020) with OrthoANIu have revealed 50 phages with genomic ANI 53.8\%-97.8\% compared to AN14 (Fig. 5), which can be considered as relatives to phage AN14. The proteomic tree clusters Yuavirus phages infecting Pseudomonas (including AN14) as a distinct group (Fig. 5). According to the formal 95\% ANI cutoff for the species definition among prokaryotes (Konstantinidis and Tiedje, 2005; Varghese et al., 2015) that is usually applied to the phages, most representatives of Yuavirus are the strains (phage races) within the same species (Fig. 5). 

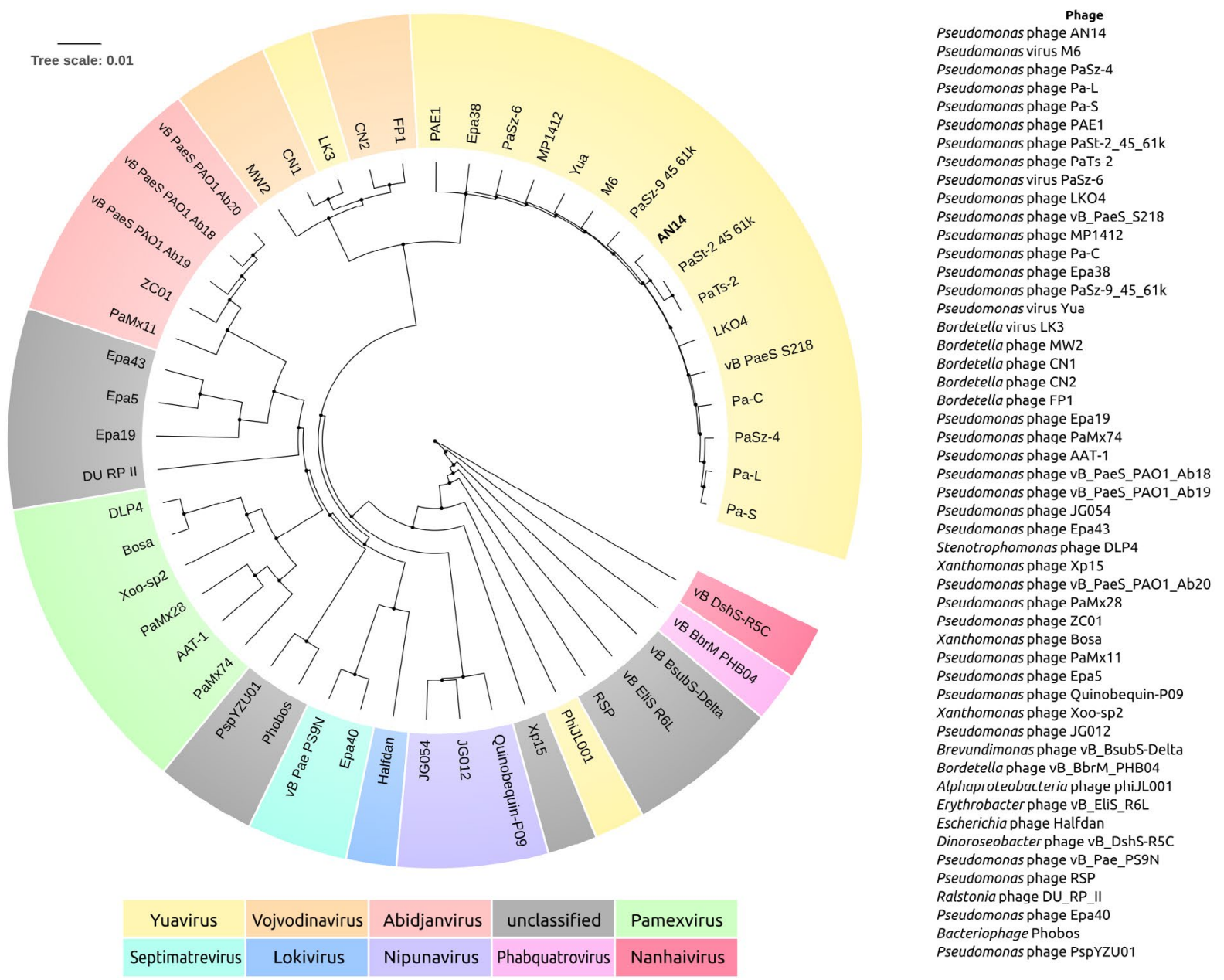

Fig.5. Proteome UPGMA tree constructed with BPGA pipeline (left diagram) and table of ANI values compared to AN14, calculated with orthoANIu (right). The tree scale bars show estimated substitutions per site.

Terminase is one of the most conserved protein complexes encoded in bacteriophage genomes, and it has been frequently used for taxonomic grouping of phages (Smith et al., 2013). In order to construct a consistent taxonomy and phylogenetic positioning of phage AN14, we performed a BLAST search using the terminase large subunit protein sequences and GenBank phage database, and constructed a list of phages belonging to the taxa, representatives of which were found with $\mathrm{E}$-value $<10^{-8}$. The maximum likelihood (ML) phylogeny of terminase large subunit protein sequence (Fig. 6) clearly showed the affiliation of AN14 with the Yuavirus clade. The tree groups 16 Pseudomonas phages into a distinct clade and points to Vojvodinavirus as the genus closest evolutionally. These phages are listed in Table 2. Contrary to the current ICTV classification, Alphaprotebacteria phage $\varphi \mathrm{JL} 001$ (AY576273) (Lohr et al., 2005) seems to belong to a group comparatively distant from Yuavirus, and Bordetella virus LK3 (KX961385) belongs to the genus Vojvodinavirus. These conclusions are in the agreement with proteome clustering, ANI calculations, genome sequence comparison among 10 AN14-related phage genomes, and ML phylogeny based on 39 core proteins concatenated sequences (Fig. 5, Fig. 6).

The terminase phylogeny points to Pseudomonas phages of the Abijanvirus genus as the next closest group to Vojvodinavirus. More distant relatives also represent the phages of gram-negative bacteria, mostly Pseudomonas spp. (Fig. 6). Phylogenetic analysis suggests that Alphaprotebacteria phage $\varphi \mathrm{JL001}$ terminase have diverged from the common clade comprising Yuavirus and related groups even earlier than the divergence to genera Stenhofvirus, Pamexvirus, Abijanvirus and Vojvodinavirus occurred.

\subsection{Yuavirus pan-genome and pan-proteome features}

The pan-genome analysis as well as ANI data demonstrated the high similarity of Pseudomonas Yuavirus phages. 55 core protein clusters (cut-off 0.5 ) have representatives in genomes of all Yuavirus phages infecting Pseudomonas. According to COG classification, about $37 \%$ of Yuavirus core proteome can be associates with the replication, recombination and repair protein category (L), $25 \%$ falls into the nucleotide transport and metabolism (F) category, $25 \%$ of core proteome belongs to the transcription $(\mathrm{K})$ category, and about $91 \%$ of accessory proteome falls into the signal transduction mechanisms (T) category (Fig. 7). The analysis of Yuavirus proteins revealed the presence of homing endonucleases in some genomes (Bae et al., 2012; Dyson et al., 2016). 


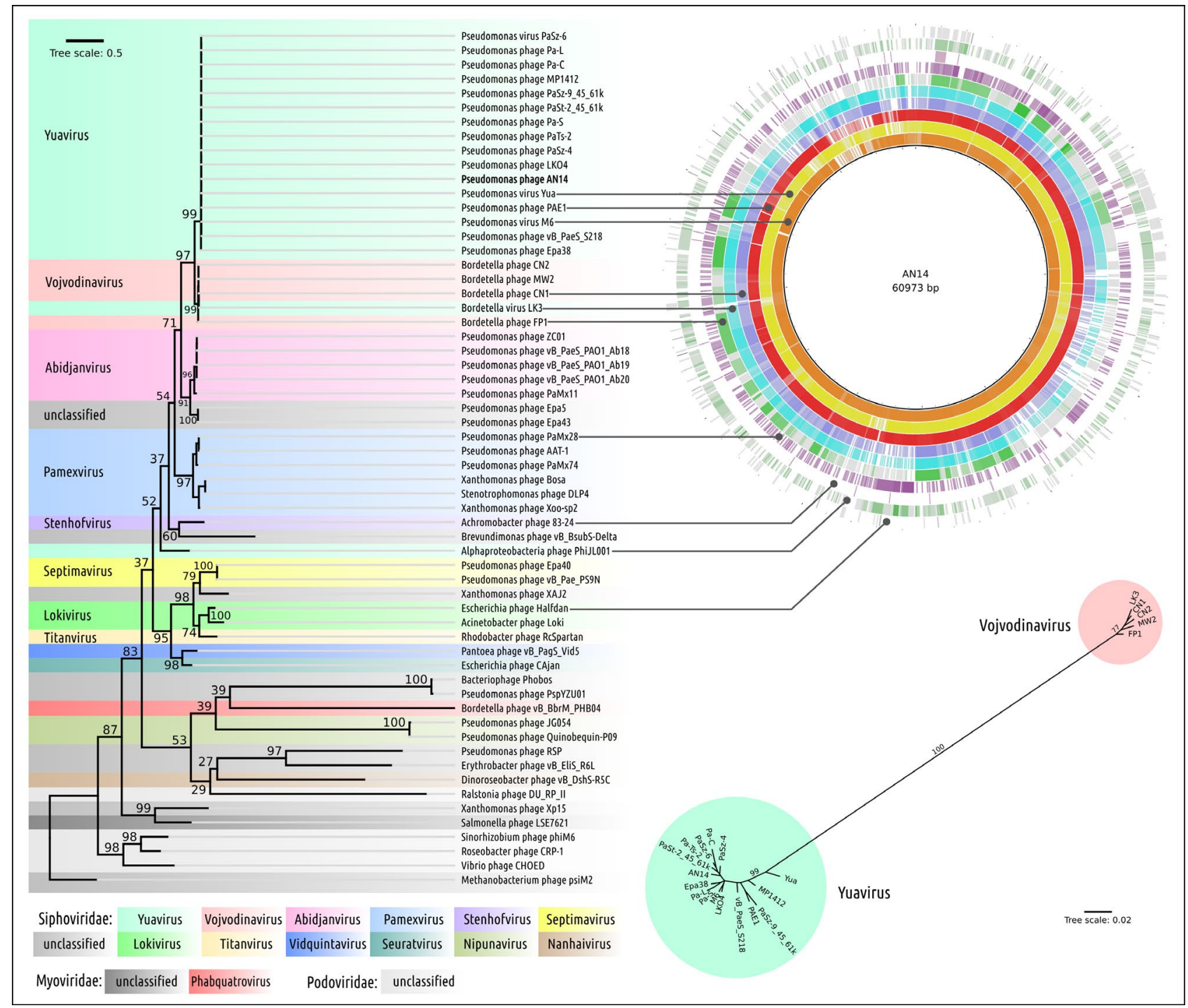

Fig.6. Best-scoring tree found by ML search with RAxML based on the terminase large subunit protein sequences (left figure), genome sequence comparison among 10 AN14-related phage genomes exhibiting co-linearity detected by TBLASTX (upper-right circular diagram), and best-scoring tree found by ML search with RAxML based on 39 core proteins concatenated sequences (lower-right figure). Bootstrap support values are shown near their branching as a percentage of 1000 replicates. The tree scale bars show estimated substitutions per site.

The 39 concatenated protein phylogeny (Fig. 6) testifies that Pseudomonas phage YuA have diverged first among 16 Pseudomonas Yuavirus phages. Pangenome analysis demonstrates that phage YuA shares the least number of common proteins with other 15 phages of this group. Meanwhile, Pseudomonas phage AN14 seems to be evolutionary closer to phages M6, PaTs-2_45_61k and PaTs-2 (Fig. 7, Table 2) and contains no genes absent in the genomes of Yuavirus phages.

\subsection{Infection cycle of Yuavirus phages}

Previously, it was shown experimentally that Pseudomonas phage YuA (Ceyssens et al., 2008) and Bordetella phages CN1, FP2, LK3 and MW2 (Petrovic et al., 2017), closely related to AN14, infect their hosts through a lytic cycle only. At least, the lysogenic behavior has not been observed, and we could not find any prophage sequences resembling the AN14 genome within the published Pseudomonas genomes with the BLAST search. More distantly related phage $\varphi J L 001$ that infects an uncharacterized marine alphaproteobacterium, JL001, was reported to be a temperate phage, but any stable lysogens were not detected as well. While induction of $\varphi J L 001$ and the homoimmunity characteristics of $\varphi \mathrm{JL} 001$ resemble true lysogeny, the phage $\varphi \mathrm{JL} 001$ genome does not integrate into host cellular replicons, possibly demonstrating a case of pseudolysogeny (Lohr et al., 2005).

The main rationale to state a possible temperate lifestyle of Yuavirus phages is the presence of the putative conserved integrase gene (g27 in case of AN14) and the repressor (g26) in the genome. However, the homologs of AN14 gp27, annotated in $\varphi \mathrm{JL} 001$, YuA, M6 and other related phages as integrases, may perform a different function not related to lysogeny. Alternatively, it may be attributed as DNA primase/polymerase belonging to AEP-primases. This proposition arises from HMM-HMM search and 3D homologous modelling (Fig. 8). The comparison with the closest structural homologs reveals that the simulated structure of AN14 gp27 contains two domains. $\mathrm{N}$-terminal domain may possess the priming 
activity, and the larger C-terminal domain possesses the helicase activity, supposedly belonging to the helicase Superfamily 3. According to HMM-HMM searches conducted with HHpred and Pyre2 server, the RepB' protein of the broad-host-range plasmid RSF1010 (Geibel et al., 2009) is the closest structural homolog of the AN14 DNA primase priming domain ( $>99 \%$ probability), and the helicase domain of the deep-sea vent phage NrS-1 polymerase (Chen et al., 2020) seems to be the closest known structural homolog of the AN14 helicase domain. Enzymes of this group may be involved to DNA reparation processes (Guilliam et al., 2015). Putative $\lambda$-like repressor encoded in AN14, as well as its homolog in $\varphi \mathrm{JL001}$, might hypothetically participate in the homoimmunity formation observed in the course at $\varphi \mathrm{JL001}$ infection (Lohr et al., 2005). Repressor in phage $\lambda$ was shown to prevent the superinfection (Fogg et al., 2010).

Undoubtedly, this hypothesis requires a future experimental proof. However, it gives a reasonable explanation for the long-disputed contradiction between the presence of predicted lysogeny genetic apparatus, and lysogeny never observed experimentally for Yuavirus phages.

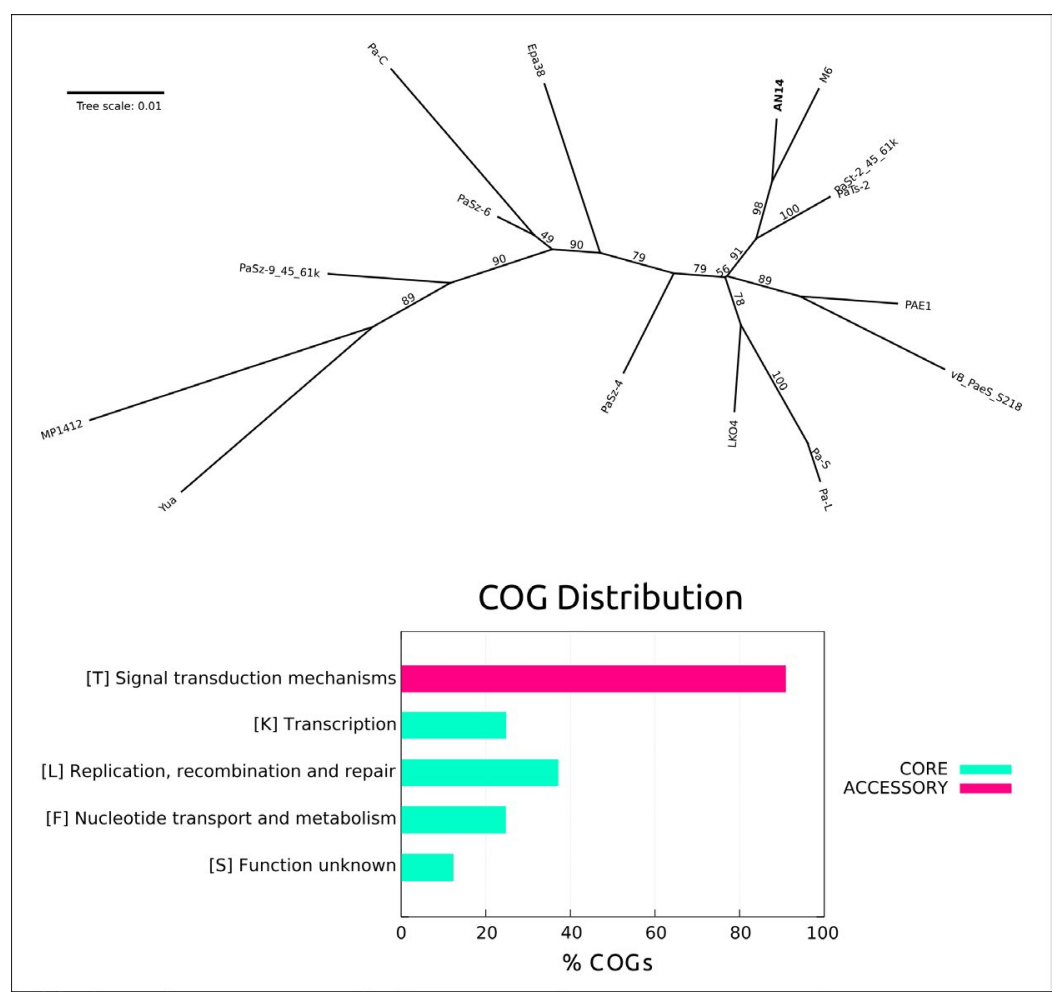

Fig.7. Best-scoring tree found by maximum likelihood (ML) search with RAxML based on 55 core proteins concatenated sequences and COG distribution diagram of Yuavirus pan-proteome constructed with BPGA pipeline. Bootstrap support values are shown near their tree branching as a percentage of 1000 replicates. The tree scale bars show estimated substitutions per site.

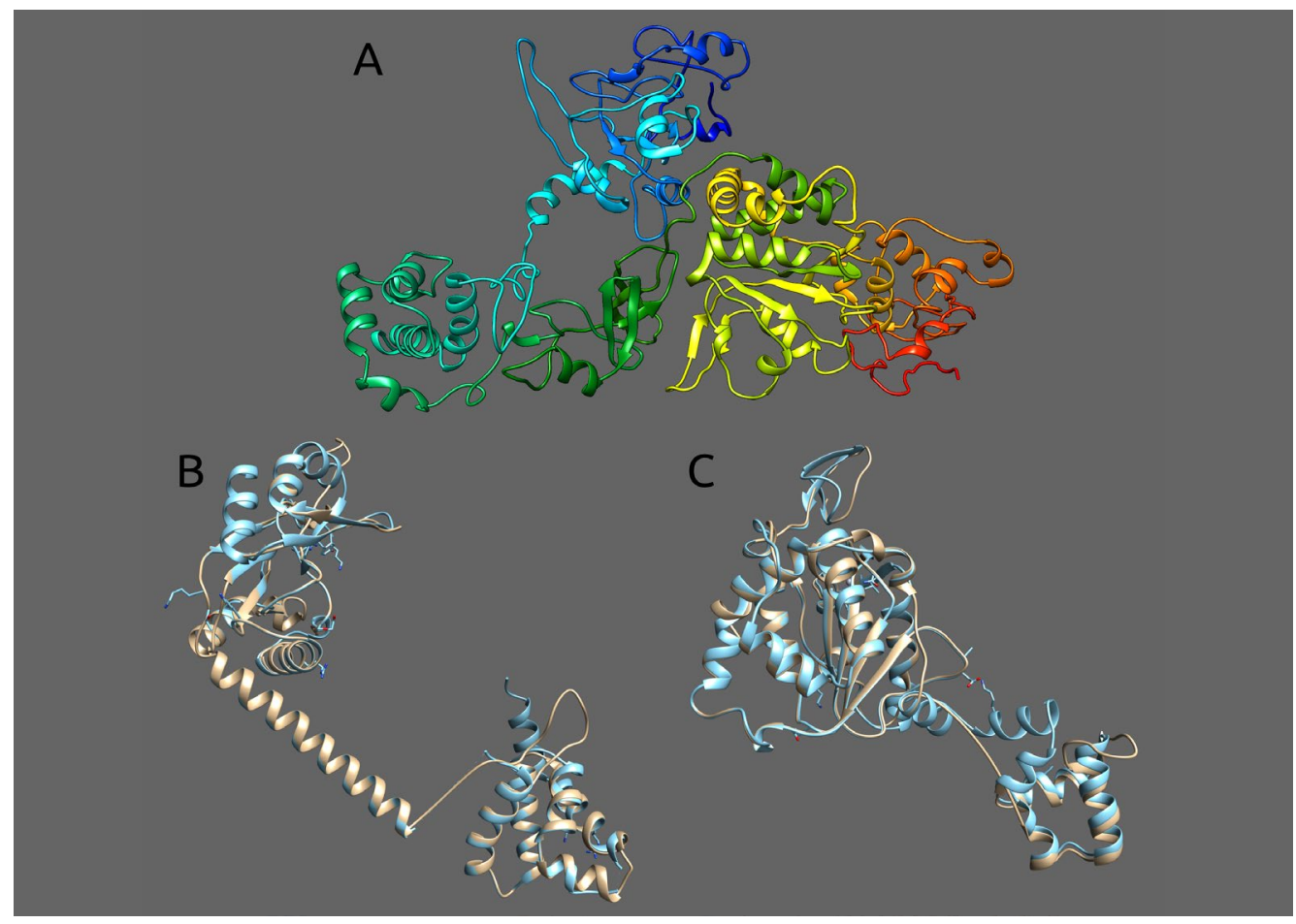

Fig.8. 3D structure homology modelling of the AN14 DNA primase (gp27). (A) Final structure obtained by Phyre2 server (http://www.sbg.bio.ic.ac.uk/phyre2). The model is colored based on a rainbow gradient scheme, where the N-terminus of the polypeptide chain is colored blue and the C-terminus is colored red. (B) 3D-alignment of obtained AN14 DNA primase protein $\mathrm{N}$-terminal domain protein model (colored pale brown) with best-fitting model (PDB entry 3H20 of crystal structure of primase RepB' from plasmid RSF1010, colored blue) made with UCSF Chimera. (C) 3D-alignment of obtained AN14 DNA primase protein C-terminal domain protein model (colored pale brown) with best-fitting model (PDB entry 6K9C of crystal structure of NrS-1 C terminal region from Nitratiruptor phage NrS-1, colored blue) made with UCSF Chimera. 
Table 2. Genomic features of Yuavirus phages

\begin{tabular}{|c|c|c|c|c|c|c|c|c|}
\hline Phage & $\begin{array}{c}\text { NCBI } \\
\text { accession \# } \\
\end{array}$ & Year & Location & Source & Host strain & $\begin{array}{c}\text { Genome, } \\
\text { bp }\end{array}$ & ORF \# & Reference \\
\hline AN14 & KX198613 & 2010 & Russia & River water & PAO1 & 60973 & 86 & This work \\
\hline YuA & AM749441 & 2008 & Russia & Pond water & PAO1 & 58663 & 77 & $\begin{array}{c}\text { (Ceyssens et al., } \\
\text { 2008) }\end{array}$ \\
\hline M6 & DQ163916 & 2006 & Canada & N/A & $\mathrm{N} / \mathrm{A}$ & 59446 & $\mathrm{~N} / \mathrm{A}$ & $\begin{array}{c}\text { (Kwan et al., } \\
\text { 2006) }\end{array}$ \\
\hline LKO4 & KC758116 & 2008 & Greece & N/A & N/A & 61818 & 77 & $\begin{array}{c}\text { Direct } \\
\text { submission }\end{array}$ \\
\hline MP1412 & JX131330 & 2011 & Korea & N/A & PAO1 & 61167 & 77 & $\begin{array}{l}\text { (Bae et al., } \\
\text { 2012) }\end{array}$ \\
\hline PAE1 & KT734862 & 2015 & Australia & Sewage & PAO9505 & 62181 & 88 & $\begin{array}{c}\text { (Dyson et al., } \\
2016 \text { ) }\end{array}$ \\
\hline vB_PaeS_S218 & MF490239 & 2015 & Italy & N/A & $\mathrm{N} / \mathrm{A}$ & 61680 & 86 & $\begin{array}{c}\text { Direct } \\
\text { submission }\end{array}$ \\
\hline PaTs-2 & MH791403 & 2017 & China & Freshwater & PAO1 & 61820 & $90 *$ & $\begin{array}{c}\text { Direct } \\
\text { submission }\end{array}$ \\
\hline $\mathrm{PaSz}-4$ & MH791406 & 2017 & China & River water & PAO1 & 61942 & $90 *$ & $\begin{array}{c}\text { Direct } \\
\text { submission }\end{array}$ \\
\hline $\mathrm{Pa}-\mathrm{C}$ & MN871456 & 2017 & China & Sea water & PAO1 & 60633 & $87 *$ & $\begin{array}{c}\text { Direct } \\
\text { submission }\end{array}$ \\
\hline Pa-L & MN871463 & 2017 & China & Sea water & PAO1 & 61641 & $87 *$ & $\begin{array}{c}\text { Direct } \\
\text { submission }\end{array}$ \\
\hline $\mathrm{Pa}-\mathrm{S}$ & MN871469 & 2017 & China & Sea water & PAO1 & 61639 & $91 *$ & $\begin{array}{c}\text { Direct } \\
\text { submission }\end{array}$ \\
\hline PaSz-6 & MN871482 & 2017 & China & Sea water & PAO1 & 54656 & $77 *$ & $\begin{array}{c}\text { Direct } \\
\text { submission }\end{array}$ \\
\hline PaSt-2_45_61k & MN871478 & 2017 & China & Sea water & PAO1 & 61865 & $90 *$ & $\begin{array}{c}\text { Direct } \\
\text { submission }\end{array}$ \\
\hline PaSz-9_45_61k & MN871486 & 2017 & China & Sea water & PAO1 & 61157 & $84 *$ & $\begin{array}{c}\text { Direct } \\
\text { submission }\end{array}$ \\
\hline Epa38 & MT118302 & 2020 & USA & N/A & N/A & 61775 & 89 & $\begin{array}{c}\text { Direct } \\
\text { submission }\end{array}$ \\
\hline Bordetella virus LK3 & KX961385 & 2013 & Serbia & $\mathrm{N} / \mathrm{A}$ & ATCC 10580 & 59831 & 79 & $\begin{array}{c}\text { Direct } \\
\text { submission }\end{array}$ \\
\hline $\begin{array}{l}\text { Alpha-proteobacteria } \\
\text { phage PhiJL001 }\end{array}$ & AY576273 & 2005 & USA & $\mathrm{N} / \mathrm{A}$ & $\mathrm{N} / \mathrm{A}$ & 63649 & 91 & $\begin{array}{l}\text { (Lohr et al., } \\
\text { 2005) }\end{array}$ \\
\hline
\end{tabular}

N/A - data not available

* Genomic annotations were performed in the course of this work. Annotation tables are available upon request from the Authors

\section{Discussion}

A group of Pseudomonas-infecting siphoviruses with an elongated capsid was discovered in mid-2000's. The first sequenced representative of this group was phage M6 (Kwan et al., 2006). However the phage YuA with comprehensively studied genomics and proteomics (Ceyssens et al., 2008) was chosen as a type phage for the recently established genus Yuavirus. In the past decade numerous Yuavirus phages were isolated from different habitats (sewage, fresh and sea water reservoirs) in Eurasia, America and Australia (Table 2). An isolation of AN14 from the ecosystem of Lake Baikal further spreads the known geography of Yuavirus phages. Genomics of most described phages of this group are very similar (Fig. 5, Fig. 6), and phage AN14 shares all morphological, biological and genomic features of Yuavirus. The representatives of this genus are perfectly accommodated to their bacterial host and possess a complicated apparatus of nucleotide modification and reparation. This may be a possible reason for slow evolution and low genomic diversity within studied YuA-like viruses.

Reannotation of AN14 genome using modern bioinformatic approaches yielded the attribution of putative functions of most phage proteins. Application of phylogenetic and pan-genomic analysis enabled us to refine composition of the genus Yuavirus. We suggest that the host range of Yuavirus is limited to Pseudomonas sp., similar to the related genus Vojvodinavirus including Bordetella phages only. The phages previously attributed to this group are distant evolutionally enough to be considered as members of other genera. 
Finally, we hypothesize the alternative function of the protein conserved among all Yuavirus phages including AN14. Predicted integrase suggesting the possibility of lysogenic behavior may be the primase/ helicase performing a different metabolic role, and, therefore, YuA-like phages possess strictly lytic infection cycle. After an experimental proof this suggestion may be important for the choice of Yuavirus phages for phage therapy purposes.

\section{Acknowledgments}

This study was carried out under the State Task No. 0345-2019-0003 (AAAA-A16-116122110061-6) "Microbial and Viral Communities in the Biofilms of Freshwater Ecosystems: Taxonomic Diversity, Functional Characteristics, and Biotechnological Potential". The microscopic studies were performed in the Instrument Center «Electron Microscopy» affiliated in the Shared Facilities for Physical and Chemical Research «Ultramicroanalysis» based in Limnological Institute of the Siberian Branch of the RAS.

\section{References}

Adams M. 1959. Bacteriophages. New York: Interscience Publishers.

Alikhan N.F., Petty N.K., Ben Zakour N.L. et al. 2011. BLAST Ring Image Generator (BRIG): simple prokaryote genome comparisons. BMC Genomics 12. DOI: 10.1186/1471-2164-12-402

Bae H.-W., Chung I.-Y., Sim N. et al. 2012. Complete genome sequence of Pseudomonas aeruginosa siphophage MP1412. Journal of Virology 86. DOI: 10.1128/JVI.01403-12

Berry J., Summer E.J., Struck D.K. et al. 2008. The final step in the phage infection cycle: the Rz and Rz1 lysis proteins link the inner and outer membranes. Molecular Microbiology 70: 341-351. DOI: 10.1111/j.1365-2958.2008.06408.x

Capella-Gutiérrez S., Silla-Martínez J.M., Gabaldón T. 2009. trimAl: a tool for automated alignment trimming in large-scale phylogenetic analyses. Bioinformatics 25: 19721973. DOI: $10.1093 /$ bioinformatics/btp348

Ceyssens P.J., Mesyanzhinov V., Sykilinda N. et al. 2008. The genome and structural proteome of YuA, a new Pseudomonas aeruginosa phage resembling M6. Journal of Bacteriology 190: 1429-1435. DOI: 10.1128/JB.01441-07

Chaudhari N.M., Gupta V.K., Dutta C. 2016. BPGA-an ultra-fast pan-genome analysis pipeline. Scientific Reports 6. DOI: $10.1038 /$ srep24373

Chen X., Su S., Chen Y. et al. 2020. Structural studies reveal a ring-shaped architecture of deep-sea vent phage NrS-1 polymerase. Nucleic Acids Research 48: 3343-3355. DOI: $10.1093 /$ nar/gkaa071

Colombet J., Robin A., Lavie L. et al. 2007. Virioplankton "pegylation": use of PEG (polyethylene glycol) to concentrate and purify viruses in pelagic ecosystems. Journal of Microbiology Methods 71: 212-219. DOI: 10.1016/j. mimet.2007.08.012

Delcher A.L., Harmon D., Kasif S. et al. 1999. Improved microbial gene identification with GLIMMER. Nucleic Acids Research 27: 4636-4641. DOI: 10.1093/nar/27.23.4636

Dyson Z.A., Seviour R.J., Tucci J. et al. 2016. Genome sequences of Pseudomonas oryzihabitans phage POR1 and Pseudomonas aeruginosa phage PAE1. Genome Announcement 4. DOI: $10.1128 /$ genomeA.01515-15
Fogg P.C.M., Allison H.E., Saunders J.R. et al. 2010. Bacteriophage Lambda: a paradigm revisited. Journal of Virology 84: 6876-6879. DOI: 10.1128/jvi.02177-09

Geibel S., Banchenko S., Engel M. et al. 2009. Structure and function of primase RepB' encoded by broad-host-range plasmid RSF1010 that replicates exclusively in leading-strand mode. Proceedings of the National Academy of Sciences of the United States of America 106: 7810-7815. DOI: 10.1073/ pnas.0902910106

Goddard T.D., Huang C.C., Meng E.C. et al. 2018. UCSF ChimeraX: meeting modern challenges in visualization and analysis. Protein Science 27: 14-25. DOI: 10.1002/pro.3235

Guilliam T.A., Keen B.A., Brissett N.C. et al. 2015. Primase-polymerases are a functionally diverse superfamily of replication and repair enzymes. Nucleic Acids Research 43: 6651-6664. DOI: 10.1093/nar/gkv625

Hyatt D., Chen G.-L., Locascio P.F. et al. 2010. Prodigal: prokaryotic gene recognition and translation initiation site identification. BMC Bioinformatics 11. DOI: 10.1186/1471-2105-11-119

Katoh K., Misawa K., Kuma K. et al. 2002. MAFFT: a novel method for rapid multiple sequence alignment based on fast Fourier transform. Nucleic Acids Research 30: 30593066. DOI: $10.1093 /$ nar/gkf436

Kelley L.A., Mezulis S., Yates C.M. et al. 2015. The Phyre2 web portal for protein modeling, prediction and analysis. Nature Protocols 10: 845-858. DOI: 10.1038/nprot.2015.053

Kongari R., Rajaure M., Cahill J. et al. 2018. Phage spanins: diversity, topological dynamics and gene convergence. BMC Bioinformatics 19. DOI: 10.1186/s12859-018-2342-8

Konstantinidis K.T., Tiedje J.M. 2005. Genomic insights that advance the species definition for prokaryotes. Proceedings of the National Academy of Sciences of the United States of America 102: 2567-2572. DOI: 10.1073/ pnas.0409727102

Kumar S., Tamura K., Nei M. 1994. MEGA: Molecular Evolutionary Genetics Analysis software for microcomputers. Bioinformatics 10: 189-191. DOI: 10.1093/ bioinformatics/10.2.189

Kwan T., Liu J., Dubow M. et al. 2006. Comparative genomic analysis of 18 Pseudomonas aeruginos a bacteriophages. Journal of Bacteriology 188: 1184-1187. DOI: 10.1128/ JB.188.3.1184-1187.2006

Lee I., Kim Y.O., Park S.C. et al. 2016. OrthoANI: an improved algorithm and software for calculating average nucleotide identity. International Journal of Systematic and Evolutionary Microbiology 66: 1100-1103. DOI: 10.1099/ ijsem. 0.000760

Lee Y.-J., Dai N., Walsh S.E. et al. 2018. Identification and biosynthesis of thymidine hypermodifications in the genomic DNA of widespread bacterial viruses. Proceedings of the National Academy of Sciences of the United States of America 15: 3116-3125. DOI: 10.1073/pnas.1714812115

Lohr J.E., Chen F., Hill R.T. 2005. Genomic analysis of bacteriophage $\Phi J L 001$ : insights into its interaction with a sponge-associated alpha-proteobacterium. Applied and Environmental Microbiology 71: 1598-1609. DOI: 10.1128/ AEM.71.3.1598-1609.2005

Petrovic A., Kostanjsek R., Rakhely G. et al. 2017. The first Siphoviridae family bacteriophages infecting Bordetella bronchiseptica isolated from environment. Microbial Ecology 73: 368-377. DOI: $10.1007 / \mathrm{s} 00248-016-0847-0$

Sayers E.W., Cavanaugh M., Clark K. et al. 2019. GenBank. Nucleic Acids Research 47: 94-99. DOI: 10.1093/nar/gky989

Schattner P., Brooks A.N., Lowe T.M. 2005. The tRNAscan-SE, snoscan and snoGPS web servers for the detection of tRNAs and snoRNAs. Nucleic Acids Research 33: W686-W689. DOI: 10.1093/nar/gki366

Seemann T. 2014. Prokka: rapid prokaryotic genome 
annotation. Bioinformatics 30: 2068-2069. DOI: 10.1093/ bioinformatics/btu153

Simm R., Morr M., Kader A. et al. 2004. GGDEF and EAL domains inversely regulate cyclic di-GMP levels and transition from sessility to motility. Molecular Microbiology 53: 11231134. DOI: 10.1111/j.1365-2958.2004.04206.x

Smith K.C., Castro-Nallar E., Fisher J.N.B. et al. 2013. Phage cluster relationships identified through single gene analysis. BMC Genomics 14. DOI: 10.1186/1471-2164-14-410

Sokolova O.S., Filchikov M.V., Pechnikova E.V. et al. 2010. Structure and virulence of Pseudomonas aeruginosa Bacteriophages. Microscopy and Microanalysis 16: 10901091. DOI: $10.1017 /$ S1431927610059994

Spoerel N., Herrlich P., Bickle T.A. 1979. A novel bacteriophage defence mechanism: the anti-restriction protein. Nature 278: 30-34. DOI: $10.1038 / 278030 \mathrm{a} 0$

Stamatakis A. 2014. RAxML version 8: a tool for phylogenetic analysis and post-analysis of large phylogenies. Bioinformatics 30: 1312-1313. DOI: 10.1093/bioinformatics/ btu033

Varghese N.J., Mukherjee S., Ivanova N. et al. 2015. Microbial species delineation using whole genome sequences. Nucleic Acids Research 43: 6761-6771. DOI: 0.1093/nar/ gkv657 